\title{
Rethinking Margin of Stability: Incorporating Step-To-Step Regulation to Resolve the Paradox
}

\author{
Meghan Kazanski ${ }^{\text {a, }}$, Joseph P. Cusumano ${ }^{\text {b }}$, Jonathan B. Dingwell ${ }^{\text {a }}$ \\ ${ }^{a}$ Department of Kinesiology, The Pennsylvania State University, University Park, PA 16802 USA \\ ${ }^{\mathrm{b}}$ Department of Engineering Science \& Mechanics, The Pennsylvania State University, University Park, PA \\ 16802 USA \\ *mek79@psu.edu
}

\begin{abstract}
Maintaining frontal-plane stability is a major objective of human walking. Derived from inverted pendulum dynamics, the mediolateral Margin of Stability $\left(M o S_{M L}\right)$ is frequently used to measure people's frontal-plane stability on average. However, typical $M o S_{M L}$-based analyses deliver paradoxical interpretations of stability status. To address mediolateral stability using $M o S_{M L}$, we must first resolve this paradox. Here, we developed a novel framework that unifies the wellestablished inverted pendulum model with Goal-Equivalent Manifold (GEM)-based analyses to assess how humans regulate step-to-step balance dynamics to maintain mediolateral stability. We quantified the extent to which people corrected fluctuations in mediolateral center-of-mass state relative to a $M o S_{M L}$-defined candidate stability GEM in the inverted pendulum phase plane. Participants' variability and step-to-step correction of tangent and perpendicular deviations from the candidate stability GEM demonstrate that regulation of balance dynamics involves more than simply trying to execute a constant- $M o S_{M L}$ balance control strategy. Participants adapted these step-to-step corrections to mediolateral sensory and mechanical perturbations. How participants regulated mediolateral foot placement strongly predicted how they regulated center-of-mass state fluctuations, suggesting that regulation of center-of-mass state occurs as a biomechanical consequence of foot placement regulation. We introduce the Probability of Instability $(P o I)$, a convenient statistic that accounts for step-to-step variance to properly predict instability likelihood on any given future step. Participants increased lateral PoI when destabilized, as expected. These lateral PoI indicated an increased risk of lateral instability, despite larger (i.e., more stable) average $M o S_{M L}$. PoI thereby explicitly predicts instability risk to decisively resolve the existing paradox that arises from conventional $M o S_{M L}$ implementations.
\end{abstract}

\section{INTRODUCTION}

Falls are a leading cause of injury among adults of all ages (Cho et al., 2021), but particularly older adults (CDC). Most people fall while walking (Heijnen and Rietdyk, 2016), and walking humans are intrinsically less stable mediolaterally (Kuo, 1999; McAndrew Young et al., 2012). Consequently, sideways falls are particularly common (Crenshaw et al., 2017) and injurious (Kannus et al., 2006; Yang et al., 2020) in older adults. Generally, humans prevent or overcome mediolateral instability by adjusting center-of-mass (CoM) dynamics and foot placement (Bruijn and van Dieën, 2018). However, we still lack a coherent framework to describe how humans regulate their movements from step-to-step to maintain mediolateral stability.

The inverted-pendulum model (Kuo, 2007; McGeer, 1990), applied to the frontal-plane during walking, represents the simplest-possible conceptualization of the mediolateral balance problem. From this model, the mediolateral Margin of Stability, $\mathrm{MoS}_{M L}$, (Hof et al., 2005) provides a measure of instantaneous frontal-plane mechanical stability, quantified using only CoM state and base-of-support dimensions at any time instant during each step (Fig. 1). Any value of $0 \leq M o S_{M L} \leq w_{\text {foot }}$ indicates intrinsic mediolateral stability. Any $M o S_{M L}$ outside of this inequality indicates intrinsic mediolateral instability, for which a sideways fall must be arrested by active rebalancing mechanisms not captured by the inverted pendulum model (e.g., by invoking a trunk inertia strategy or taking a step) (Otten, 1999). Thus, $M o S_{M L}$ defines the biomechanical balance requirements that must be met within any individual step and directly quantifies (as a physical distance) how close a system is to becoming mediolaterally unstable at any instant. A larger instantaneous $M o S_{M L}$ in a given direction should indicate greater intrinsic frontal plane stability in that direction. 


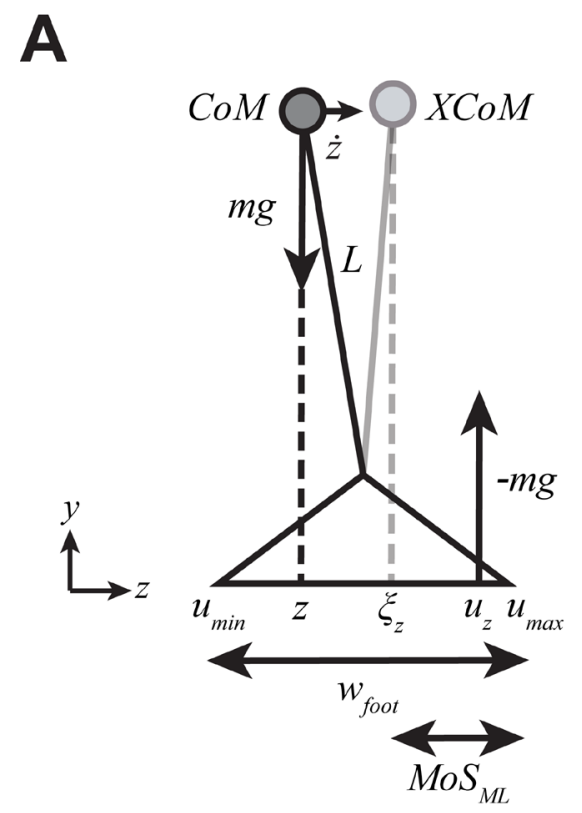

B

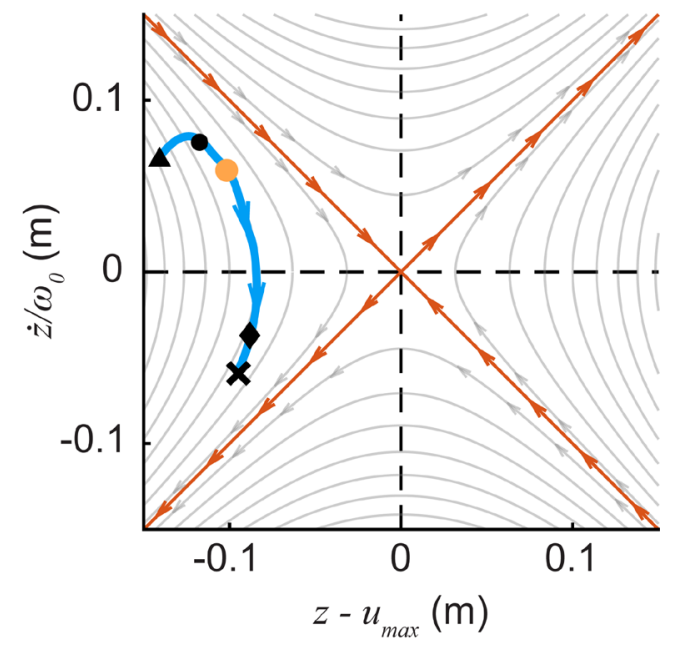

Figure 1 - A) The inverted pendulum model, applied to the frontal plane, defines mediolateral CoM dynamics $(z, \dot{z})$ throughout each single-stance phase of gait (adapted from (Hof et al., 2005)). Motion is modeled as a point mass $m$ located at the body CoM, attached a distance $L$ from the ankle joint of the base-of-support (stance foot) of width $w_{\text {foot }}$. The mediolateral center of pressure $u_{z}$ is the point where the ground reaction force is applied between $u_{\min }$ (medial bound) and $u_{\max }$ (lateral bound). The mediolateral margin of stability $\left(M o S_{M L}\right)$ is the medial-to-lateral distance that the velocity-adjusted ('extrapolated') center of mass $\left(\xi_{z}=z+\right.$ $\left.\dot{z} / \omega_{0}\right)$ can travel toward $u_{\max }$ (Eq. (1)) before active intervention is required to arrest a lateral fall. B) The phase portrait of the inverted pendulum model's dynamics. The vertical axis $\left(\dot{z} / \omega_{0}\right)$ is the mediolateral CoM velocity scaled by the pendulum eigenfrequency. The horizontal axis $\left(z-u_{\max }\right)$ is the mediolateral CoM position relative to the lateral base-of-support boundary. Here, the dynamics are plotted for the case where $u_{z} \approx u_{\max }=$ constant. Grey flow lines (Bottaro et al., 2005; Hof, 2008) demonstrate the intrinsic CoM dynamics of the inverted pendulum model (flow direction indicated by arrows). The blue trajectory shows the dynamics of a single representative step of human walking from heel-strike $(\boldsymbol{\Lambda})$, to contralateral toe-off (single-stance onset) $(\bullet)$ to contralateral heel-strike $(\bullet)$, until ipsilateral toe-off $(\times)$. For any given step, this trajectory has a minimum $M o S_{M L}($ yellow $\bullet$ ).

$M o S_{M L}$ is a mechanically valid measure of instantaneous mediolateral stability. However, its application to human walking becomes problematic and misleading when $M o S_{M L}$ values are averaged across steps. People exhibit larger average $M o S_{M L}$ (supposedly indicating enhanced stability) when subjected to substantial de-stabilizing perturbations (McAndrew Young et al., 2012; Selgrade et al., 2020). Likewise, multiple populations known to be substantially more fall-prone exhibit larger average $M o S_{M L}$, including older adults (Arvin et al., 2016) and persons with stroke (Tisserand et al., 2018), amputation (Hof et al., 2007), spinal cord injury (Day et al., 2012), or multiple sclerosis (Peebles et al., 2016). Paradoxically, extrinsically-destabilized and balance-impaired individuals are thereby classified as more stable mediolaterally. This paradox arises from using average values of $M o S_{M L}$ to determine mediolateral stability status.

Conversely, people do not fall "on average" - falls result from singular events (e.g., perturbations) that occur in real time (i.e., within a step). Averaging $M o S_{M L}$ (or any such similar measure) across multiple steps inherently discards critical information regarding how $M o S_{M L}$ varies from step-to-step. $M o S_{M L}$ also does not capture how people adjust their CoM dynamics and foot placements to maintain or recover balance. It is therefore imperative that we examine how humans regulate step-to-step fluctuations in these quantities to maintain $M o S_{M L}$-defined mediolateral stability across steps.

The goal-equivalent manifold (GEM) concept (Cusumano and Cesari, 2006) formulates mathematically-defined task goal functions and optimal control models to pose testable hypotheses regarding how humans regulate stepping movements to achieve walking (Dingwell et al., 2010). This framework successfully predicts how humans regulate mediolateral foot placement during unperturbed (Dingwell and Cusumano, 2019) and perturbed (Kazanski et al., 2020) walking, or when given explicit task feedback (Render et al., 2021). We should be able to apply the same GEM-based concepts to also predict how people regulate their CoM dynamics in conjunction with step-to-step foot placement to achieve specified stability objectives. Here, we demonstrate this. 
Additionally, if people indeed regulate their stepping movements to achieve specific balance goals (Bruijn and van Dieën, 2018), this regulation likely reflects the extent to which they consider instability risk at each step. In reaching tasks, humans use "risk-sensitive" control strategies (Braun et al., 2011) that seek to avoid variance per se (John et al., 2016; Nagengast et al., 2011). People indeed implement strategies that modulate instability risk during walking: e.g., to trade off mediolateral stability for effort (Dean et al., 2007) and/or maneuverability (Hsieh et al., 2018). Humans may mitigate potential risk of mediolateral instability by structuring variance of mediolateral CoM dynamics from step-to-step. Neither instantaneous nor average measures of $M o S_{M L}$ can predict risk of mediolateral instability on any future step. Here, we demonstrate that incorporating the variance of stepping dynamics yields proper measures that explicitly predict instability risk.

In this paper, we present a novel analytical framework that unifies the well-established inverted-pendulum model with GEM-based stepping regulation analyses. We use this framework to develop and test hypotheses regarding how humans maintain mediolateral stability and mitigate potential instability risk. First, we identify a candidate stability GEM in the dynamics of the inverted pendulum model, defined by the objective to maintain some constant $M o S_{M L}$ from step-to-step. We hypothesize that humans maintain balance by coordinating mediolateral CoM dynamics to achieve a constant $\operatorname{MoS}_{M L}$ (Curtze et al., 2011; Hof, 2008; Rosenblatt and Grabiner, 2010). We test this by evaluating how people regulate their CoM dynamics relative to this candidate GEM. Then, we examine how this regulation varies with age and externally-imposed balance perturbations. We then pose a complementary hypothesis that humans achieve mediolateral CoM regulation as a mechanical consequence of regulating mediolateral foot placement. Foot placement generally modulates mediolateral balance (Bruijn and van Dieën, 2018) and strongly correlates with CoM state fluctuations (Patil et al., 2019; Wang and Srinivasan, 2014). We test this by evaluating the extent to which step-to-step correction of mediolateral foot placement fluctuations (Kazanski et al., 2020) predicts correction of mediolateral CoM state fluctuations. Together, these hypotheses address how CoM states are regulated to maintain mediolateral stability, and how this regulation is achieved mechanically. Lastly, we demonstrate that these step-to-step CoM fluctuations have clear implications for mediolateral instability risk not captured by conventional $M o S_{M L}$ calculations. We instead propose a new measure of instability risk that decisively resolves the existing paradox by incorporating how step-tostep variance critically influences risk of mediolateral instability.

\section{METHODS}

\section{Participants, Protocol, and Data Processing}

Complete descriptions of participants, assessments, and experimental protocol are presented in Kazanski et al. (2020). We conducted the analyses presented here on data from the same cohort of young healthy (YH) and older healthy $(\mathrm{OH})$ adults. $\mathrm{OH}$ participants selected slightly slower preferred walking speeds and exhibited slightly slower Four-Square Step Test scores than YH adults (Table 1).

All participants provided written informed consent, as approved by The University of Texas at Austin's IRB, and had no physiological impairments or limitations that affected their walking. Participants walked in a "V-Gait" virtual reality system, (Motekforce Link, Amsterdam, Netherlands). For each participant, treadmill and visual optic flow speeds were both set to $90 \%$ of their preferred overground walking speed. Following a 3-minute treadmill-acclimation trial, participants performed three, 3-minute trials of treadmill walking for each of 3 conditions: normal walking with no perturbations (NOP), or walking with mediolateral oscillations of either the visual field (VIS) or treadmill platform (PLAT).

Here, we analyzed data from retroreflective markers placed on each heel and lateral malleolus, and four markers placed on the pelvis (additional details in (Kazanski et al., 2020)). We analyzed the latter two of three trials for each condition. Three PLA trials $(2 \mathrm{YH}, 1 \mathrm{OH})$ were not analyzed due to excessive marker dropout. Marker trajectory data were filtered $\left(2^{\text {nd }}\right.$-order, low-pass Butterworth; cutoff $\left.=6 \mathrm{~Hz}\right)$ and interpolated to $600 \mathrm{~Hz}$. Heel-strike and toe-off events were defined for each step (Zeni et al., 2008). We analyzed $N=230$ consecutive steps following the first $15 \mathrm{~s}$ of each trial. 
bioRxiv preprint doi: https://doi.org/10.1101/2021.12.01.470263; this version posted December 3, 2021. The copyright holder for this preprint

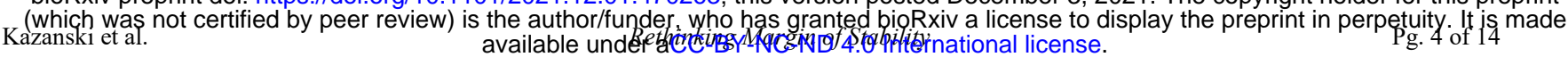

\begin{tabular}{llll}
\hline Characteristic: & Young Healthy (YH): & Older Healthy $(\mathbf{O H}):$ & p-value \\
\hline Sex [M/F] & $8 / 9$ & $7 / 10$ & N/A \\
Age [yrs] & $23.7 \pm 3.7$ & $67.5 \pm 4.9$ & $<\mathbf{0 . 0 0 1}$ \\
Body Height [m] & $1.72 \pm 0.1$ & $1.71 \pm 0.1$ & 0.772 \\
Body Mass [kg] & $64.5 \pm 12.5$ & $73.3 \pm 18.9$ & 0.118 \\
Body Mass Index [kg/m²] & $21.7 \pm 3.2$ & $24.9 \pm 5.4$ & $\mathbf{0 . 0 4 2}$ \\
Leg Length [m] & $0.89 \pm 0.05$ & $0.90 \pm 0.05$ & 0.453 \\
\hline Assessment: & & & \\
\hline Timed Up and Go [s] & $8.06 \pm 1.20$ & $8.66 \pm 1.08$ & 0.139 \\
Four Square Step Test [s] & $7.18 \pm 1.53$ & $9.07 \pm 2.07$ & $\mathbf{0 . 0 0 5}$ \\
Preferred Walking Speed [m/s] & $1.46 \pm 0.15$ & $1.35 \pm 0.14$ & $\mathbf{0 . 0 3 3}$
\end{tabular}

Table 1 - Participant characteristics and physical assessment results for Young (YH) and Older $(\mathrm{OH})$ healthy adults. For additional details, see (Kazanski et al., 2020). All values except Sex are given as mean \pm standard deviation. Two-sample t-test results (pvalues) indicating significant Age group differences are shown in bold.

\section{Mediolateral Margin of Stability}

We used the $z$-direction pelvic centroid motion to approximate continuous mediolateral CoM state $(z, \dot{z})$. We used the mediolateral position of the leading foot's lateral malleolus marker to define the lateral base-of-support boundary $\left(u_{\max }\right)$. Directly following Hof et al.'s original expression (2005), we computed the minimum mediolateral Margin of Stability $\left(M o S_{M L}\right)_{n}$ that occurred during each $n^{\text {th }}$ step:

$$
\left(M o S_{M L}\right)_{n}=\left(u_{\max }\right)_{n}-\left(z+\dot{z} / \omega_{0}\right)_{n},
$$

where $\left(z+\dot{z} / \omega_{0}\right)$ is the "extrapolated" mediolateral CoM position that accounts for CoM velocity, normalized by eigenfrequency $\omega_{0}=(g / L)^{0.5} . L$ is an equivalent pendulum length taken as $L=1.34 * l$, where $l$ is leg length measured as the vertical distance from lateral malleolus to greater trochanter (Hof et al., 2005).

For each walking trial, we extracted time series of $M o S_{M L}$ for all $n \in(1, \ldots, N)$ steps. We then computed the mean, variability (standard deviation: $\sigma$ ), and statistical persistence (DFA exponent: $\alpha$ ) (Hausdorff et al., 1995) of each $M o S_{M L}$ time series. For the DFA exponents, $\alpha>1 / 2$ indicates statistical persistence, $\alpha<1 / 2$ indicates anti-persistence, and $\alpha=1 / 2$ indicates uncorrelated data. Tightly-regulated variables typically exhibit $\alpha \approx 0.5$ (reflecting rapid correction of deviations), whereas weakly-regulated variables typically exhibit $\alpha \gg 1 / 2$ (reflecting minimal step-to-step corrections) (Dingwell and Cusumano, 2010, 2019). Here, we used the $\alpha\left(M o S_{M L}\right)$ data to evaluate whether participants' step-to-step fluctuations in $M o S_{M L}$ were consistent with tight regulation.

For each dependent measure, we performed a two-factor mixed-effects (Age $\times$ Condition) analysis of variance (ANOVA) (see Supplement for details).

\section{Candidate Stability Goal-Equivalent Manifold}

$M o S_{M L}$ (Eq. (1)) assumes human mediolateral balance is well-approximated by an inverted pendulum (Fig. 1A), the dynamics of which are fully captured by its CoM phase portrait, [z-umax,$\left.\dot{z} / \omega_{0}\right]$ (Fig. 1B) (Bottaro et al., 2005; Hof, 2008). For an inverted-pendulum with base-of-support bounds $\left[u_{\min }, u_{\max }\right]$ and width $w_{\text {foot }}=u_{\min }-u_{\max }$, lateral instability is avoided for $M o S_{M L} \geq 0$, while medial instability is avoided for $M o S_{M L} \leq w_{\text {foot }}$ (Hof et al., 2005). In the [z- $\left.u_{\max }, \dot{z} / \omega_{0}\right]$ plane, this forms a Stability region between two diagonal boundaries (Fig. 2A), wherein all combinations of $\left[\left(z-u_{\max }\right)_{n}\right.$, $\left.\left(\dot{z} / \omega_{0}\right)_{n}\right]$ achieve a mediolaterally stable $M o S_{M L}$. Mediolateral CoM dynamics outside this region indicate instability and require active intervention to regain balance.

It is thus evident that a primary task requirement for stable walking is to remain within this Stability region (Fig. $2 \mathrm{~A}$ ) for all $n$ steps in a sequence of $N$ consecutive steps. Mathematically, this is defined as maintaining combinations of $\left[\left(z-u_{\max }\right)_{n},\left(\dot{z} / \omega_{0}\right)_{n}\right]$ that achieve:

$$
\forall n \in\{1, \cdots, N\}: 0<\left(M o S_{M L}\right)_{n}<w_{\text {foot }} .
$$


A

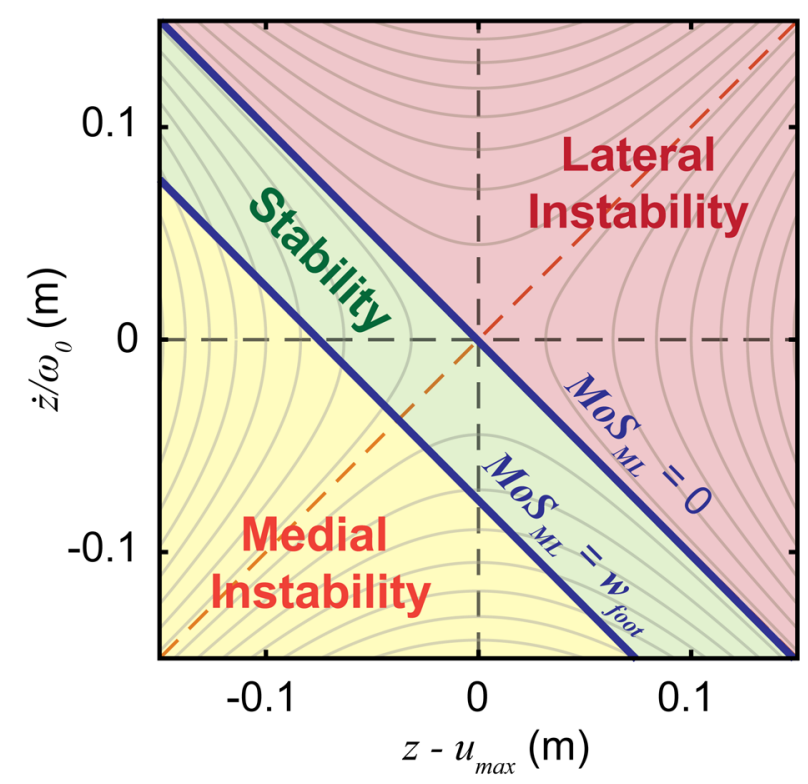

B

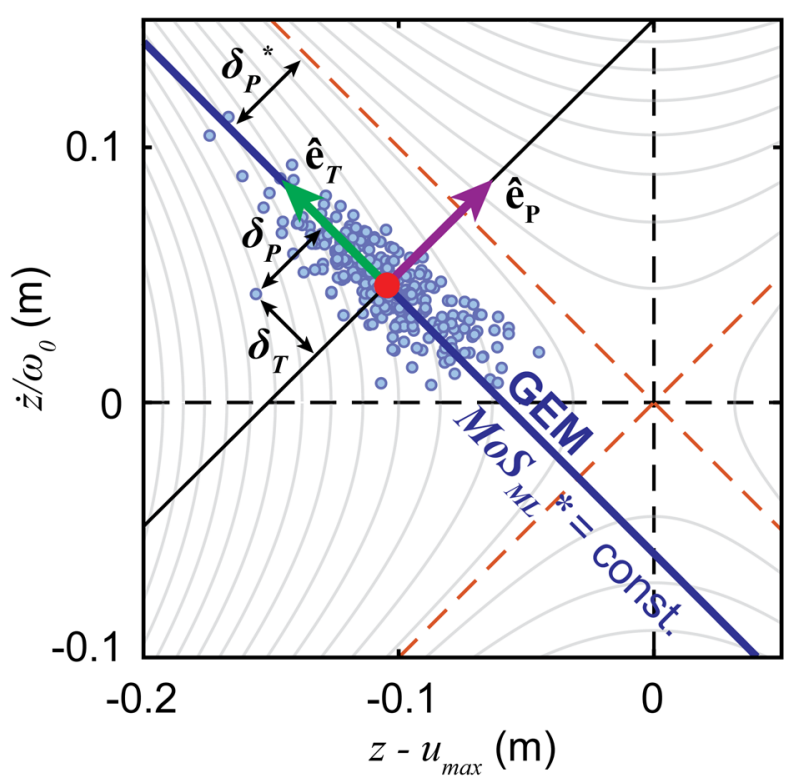

Figure 2 - (A): The mediolateral Margin of Stability $\left(M o S_{M L}\right)$ predicts distinct stability regions in the CoM phase plane $\left[z-u_{\max }\right.$, $\left.\dot{z} / \omega_{0}\right]$. With the positive $z$-direction defined as medial-to-lateral, $M o S_{M L}=0$ defines the critical threshold between the Stability (green) and Lateral Instability (red) regions. For dynamics in the Lateral Instability region, a lateral fall could result if no active intervention is executed to return the system to the Stability region. Likewise, $M o S_{M L}=w_{\text {foot }}$ defines the critical threshold between the Stability and Medial Instability (yellow) regions, wherein a medial fall could result if no active intervention is executed. (B): A candidate stability goal-equivalent manifold (GEM; diagonal blue line) in the CoM phase plane $\left[z-u_{\max }, \dot{z} / \omega_{0}\right]$. Any such diagonal line defines all combinations of CoM states that achieve the same constant $M o S_{M L}$ : i.e., $M o S_{M L}{ }^{*}$. An infinity of such GEMs exists for all possible values of $\mathrm{MoS}_{M L}{ }^{*}$ (Eq. 3). Data points (filled blue markers) represent single steps from a typical trial, each taken at the instant the mediolateral CoM state attains the minimum $M o S_{M L}$ for that step. We propose one candidate stability GEM to indicate the hypothesized task objective to maintain constant $M o S_{M L}{ }^{*}$ (Eq. 4) as the average of $\left(M o S_{M L}\right)_{n}$ across all steps for a given trial. Orthonormal basis unit vectors $\left[\hat{e}_{T}, \hat{e}_{P}\right]$ are aligned tangent and perpendicular to the stability GEM, respectively. Time series of CoM states are transformed (Eq. 5) into deviations $\left[\delta_{T}, \delta_{P}\right]$ tangent and perpendicular, respectively, to the candidate stability GEM at the mean operating point (red filled circle). Quantity $\delta_{P}{ }^{*}$ is the distance between the $M o S_{M L}{ }^{*}$ GEM and the $M o S_{M L}=0$ lateral stability bound along $\hat{e}_{P}$.

Because any sequence of $\left[\left(z-u_{\max }\right)_{n},\left(\dot{z} / \omega_{0}\right)_{n}\right]$ that satisfies Eq. (2) - and there are infinite such combinations - will achieve mediolaterally stable walking, many strategies exist to generate such feasible stepping sequences.

Here, we note that rearranging Eq. (1) as:

$$
\left(\dot{z} / \omega_{0}\right)_{n}=-\left(z-u_{\max }\right)_{n}-\left(M o S_{M L}\right)_{n}
$$

demonstrates that any constant-valued $M o S_{M L}$ yields a diagonal line in $\left[z-u_{\max }, \dot{z} / \omega_{0}\right]$. Along any such diagonal, infinite combinations of $\left[\left(z-u_{\max }\right)_{n},\left(\dot{z} / \omega_{0}\right)_{n}\right]$ achieve the same specified constant-valued $M o S_{M L}$. We thus propose that the simplest approach to satisfying Eq. (2) across multiple steps is to coordinate $n^{\text {th }}$ step CoM states to maintain some constant $M o S_{M L}{ }^{*}$, such that $0<M o S_{M L}{ }^{*}<w_{\text {foot }}$. We express this candidate stabilizing approach as the goal function (Fig. 2B):

$$
F\left[\left(z-u_{\max }\right)_{n},\left(\dot{z} / \omega_{0}\right)_{n}\right]=\left(M o S_{M L}\right)^{*}+\left[\left(z-u_{\max }\right)_{n}+\left(\dot{z} / \omega_{0}\right)_{n}\right],
$$

where the person's goal is to drive $F \rightarrow 0$. Eq. (4) defines a candidate Goal-Equivalent Manifold (GEM) for mediolateral stability as a specific diagonal line that contains all possible $\left[\left(z-u_{\max }\right)_{n},\left(\dot{z} / \omega_{0}\right)_{n}\right]$ achieving $\operatorname{MoS}_{M L}{ }^{*}$. 
Here, we test the hypothesis that humans adopt a constant- $M o S_{M L}{ }^{*}$ stability approach, adjusting $\left[\left(z-u_{\max }\right)_{n},\left(\dot{z} / \omega_{0}\right)_{n}\right]$ at each step to minimize errors relative to this candidate stability GEM.

For each trial, we defined the candidate stability GEM by $M o S_{M L}{ }^{*}=\left\langle\left(M o S_{M L}\right)_{n}\right\rangle_{N}$, where $\langle\cdot\rangle_{N}$ denotes the average over $N$ steps. Following (Dingwell et al., 2010), we defined an "operating point" on the GEM as $\left(z-u_{\max }\right)^{*}=\left\langle\left(z-u_{\max }\right)_{n}\right\rangle_{N}$ and $\left(\dot{z} / \omega_{0}\right)^{*}=\left\langle\left(\dot{z} / \omega_{0}\right)_{n}\right\rangle_{N}$. We defined deviations in $\left[\left(z-u_{\max }\right)_{n},\left(\dot{z} / \omega_{0}\right)_{n}\right]$ from this operating point as $\left(z-u_{\max }\right)_{n}^{\prime}=\left(z-u_{\max }\right)_{n}-$ $\left(z-u_{\max }\right)^{*}$ and $\left(\dot{z} / \omega_{0}\right)_{n}^{\prime}=\left(\dot{z} / \omega_{0}\right)_{n}-\left(\dot{z} / \omega_{0}\right)_{n}{ }^{*}$. We then linearly transformed $\left[\left(z-u_{\max }\right)_{n}^{\prime},\left(\dot{z} / \omega_{0}\right)_{n}^{\prime}\right]$ coordinates into $\left[\left(\delta_{T}\right)_{n},\left(\delta_{P}\right)_{n}\right]$ coordinates along GEM-specific unit vectors $\left[\hat{e}_{T}, \hat{e}_{P}\right]$ (Fig. 2B):

$$
\left[\begin{array}{l}
\left(\delta_{T}\right)_{n} \\
\left(\delta_{P}\right)_{n}
\end{array}\right]=\frac{1}{\sqrt{2}}\left[\begin{array}{cc}
-1 & 1 \\
1 & 1
\end{array}\right]\left[\begin{array}{c}
\left(z-u_{\max }\right)^{\prime} n \\
\left(\dot{z} / \omega_{0}\right)^{\prime}{ }_{n}
\end{array}\right]
$$

Deviations tangent to the GEM $\left(\delta_{T}\right)$ are "goal-equivalent," having no impact on $M o S_{M L}$. Conversely, deviations perpendicular to the GEM $\left(\delta_{P}\right)$ are "goal-relevant," having direct impact on $M o S_{M L}$. We computed time series of $\delta_{T}$ and $\delta_{P}$ for each trial. For each, we computed variability $(\sigma)$ to quantify the average deviation magnitude, and statistical persistence (DFA exponent: $\alpha$ ) to evaluate step-to-step correction of deviations. If participants adhered to the candidate stability GEM, we predicted they would: (1) reduce goal-relevant deviations from the GEM, indicated by $\sigma\left(\delta_{P}\right)<<$ $\sigma\left(\delta_{T}\right)$ and also (2) more-readily correct goal-relevant deviations, indicated by $\alpha\left(\delta_{P}\right) \approx 0.5<<\alpha\left(\delta_{T}\right)$ (Dingwell and Cusumano, 2019; Dingwell et al., 2010). For each dependent measure, we conducted three-factor and two-factor mixed-effects ANOVAs to test these predictions (see Supplement for details).

\section{Regulating Foot Placement to Maintain Mediolateral Balance}

It is not obvious that walking humans can directly correct CoM deviations from the GEM: they have no means to directly actuate their CoM. However, they do systematically directly regulate mediolateral foot placements from each step to the next (Kazanski et al., 2020). We thus hypothesized that people regulate mediolateral CoM dynamics as mechanical consequence of how they regulate step-to-step fluctuations in step width $(w)$ and lateral body position $\left(z_{B}\right)$ (as previously reported in (Kazanski et al., 2020)). To test this hypothesis, we computed pairwise Pearson correlations to assess whether, across age groups and conditions, the extent to which participants corrected step-wise deviations in mediolateral foot placement (as quantified by $\alpha(w)$ and $\alpha\left(z_{B}\right)$ ) predicted the extent to which they corrected deviations in mediolateral CoM state (as quantified by $\alpha\left(\delta_{T}\right)$ and $\alpha\left(\delta_{P}\right)$ ).

\section{Probability of Instability (PoI)}

It is evident from Fig. 2 that average values of $M o S_{M L}$ cannot predict mediolateral instability likelihood on any future step. $M o S_{M L}$ itself is an instantaneous measure of mediolateral stability (Hof et al., 2005). Because $M o S_{M L}$ does not account for step-to-step variations, averaging $M o S_{M L}$ across steps yields paradoxical findings (e.g., (Arvin et al., 2016; McAndrew et al., 2011), etc.). Here, we propose a novel measure, the Probability of Instability (PoI), to quantify intrinsic instability risk for any future step.

$\mathrm{PoI}_{L}$ measures lateral instability risk, computed as the percent likelihood that CoM dynamics from any future step will fall in the Lateral Instability region: i.e., $M o S_{M L}<0$ (Fig. 2A). Assuming a normally-distributed $M o S_{M L}$ time series:

$$
\mathrm{PoI}_{L}=\frac{1}{2}\left[1-\operatorname{erf}\left(\frac{M o S_{M L}^{*}}{\sigma\left(M o S_{M L}\right)} / \sqrt{2}\right)\right] \times 100 \%,
$$

Or equivalently, the probability that future $\delta_{P}$ will exceed the $M o S_{M L}=0$ bound along $\hat{e}_{P}$ (Fig 2B):

$$
P o I_{L}=\frac{1}{2}\left[1-\operatorname{erf}\left(\frac{\delta_{P}^{*}}{\sigma\left(\delta_{P}\right)} / \sqrt{2}\right)\right] \times 100 \%
$$

where $\delta_{P}^{*}=1 / \sqrt{2}\left(M o S_{M L}^{*}\right)$ is the distance along $\hat{e}_{P}$ between the $M o S_{M L}{ }^{*}$ GEM and $M o S_{M L}=0$ diagonals. The error function, $\operatorname{erf}(\bullet)$ (Andrews, 1998), quantifies the expected fraction of the $\delta_{P}$ distribution contained between $\pm \delta_{P} *$ along $\hat{e}_{P}$. Importantly, $P_{o I} I_{L}$ uses mediolateral CoM state variance to directly calculate a participant's lateral instability risk on any future step, no matter their average $M o S_{M L}$ (Fig. 3).

We computed $\mathrm{PoI}_{L}$ for each trial and conducted a two-factor mixed-effects (Age $\times$ Condition) ANOVA (see Supplement for details) to assess age group and condition differences. 
A

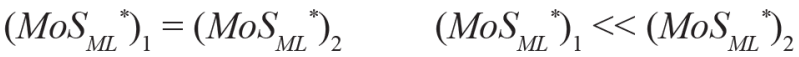

$$
\begin{aligned}
& \left(\mathrm{PoI}_{L}\right)_{1} \gg\left(\mathrm{PoI}_{L}\right)_{2} \quad\left(\mathrm{PoI}_{L}\right)_{1}=\left(\mathrm{PoI}_{L}\right)_{2}
\end{aligned}
$$

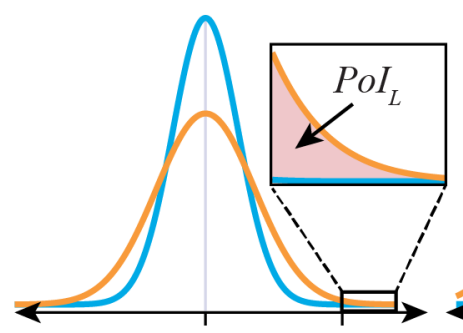

$\left(M o S_{M L}^{*}\right)_{1,2}$

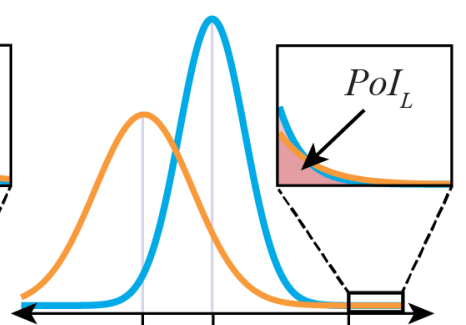

$\left(M o S_{M L}^{*}\right)_{1}\left(M o S_{M L}^{*}\right)_{2} 0$
Figure 3 - Probability of Instability $\left(P_{o} I_{L}\right)$ depends on both the mean and variance of the distribution of any given $M o S_{M L}$ time series. To demonstrate this, consider two hypothetical trial distributions in orange (1) and blue (2). In (A), distributions with the exact same means $\left(M o S_{M L}{ }^{*}\right)$ can still yield substantially different $\operatorname{PoI}_{L}$. Conversely, in (B), distributions with very different means can still yield the exact same $P_{0} I_{L}$.

\section{RESULTS}

All participants exhibited significantly larger $M o S_{M L}$ means during perturbed walking (VIS and PLAT) relative to unperturbed walking (NOP) (Fig. 4A; $\mathrm{p}<0.005$; Table S1). All participants also demonstrated significantly morevariable $M o S_{M L}$ when perturbed (Fig. 4B; $<<10^{-11}$; Table S1). Participants generally exhibited slight anti-persistence of $M o S_{M L}$ time series when perturbed (Fig. 4C; Table S1). YH adults exhibited significantly decreased DFA $\alpha$ during both VIS $\left(\mathrm{p}<10^{-4}\right)$ and PLAT $\left(\mathrm{p}<10^{-12}\right)$ perturbations. OH adults exhibited significantly decreased DFA $\alpha$ only during PLAT $\left(\mathrm{p}<10^{-7}\right)$ perturbations (Table $\left.\mathrm{S} 1\right)$. Age group effects were not significant.

Across all conditions, step-to-step mediolateral CoM dynamics were strongly elongated along the candidate constant-stability GEM (Fig. 5). Under VIS and PLAT perturbations, distribution clouds of data points appeared larger relative to NOP (Fig. 5B-C), but remained strongly elongated along the constant-stability GEM.

Across all participants and conditions, $\delta_{P}$ deviations were both significantly less variable (Fig. 6A; $\sigma\left(\delta_{P}\right)<<\sigma\left(\delta_{T}\right)$; $\mathrm{p}=3.69 \times 10^{-141}$; Table S2) and either more statistically persistent (higher $\alpha>0.5$ ) or less anti-persistent (higher $\alpha<$ $0.5)$ (Fig. 6B; $\alpha\left(\delta_{P}\right)<<\alpha\left(\delta_{T}\right) ; \mathrm{p}=1.53 \times 10^{-15}$; Table S2) than $\delta_{T}$ deviations. Both $\sigma\left(\delta_{P}\right)$ and $\sigma\left(\delta_{T}\right)$ increased significantly during perturbation conditions (Fig. 6A; all $\mathrm{p}<10^{-11}$; Table S3). Indicated by $\alpha\left(\delta_{P}\right)$ and $\alpha\left(\delta_{T}\right)$, deviations in both directions generally became either less statistically persistent or even anti-persistent during perturbation conditions (Fig. 6B; Table S2). Age group effects were not significant.
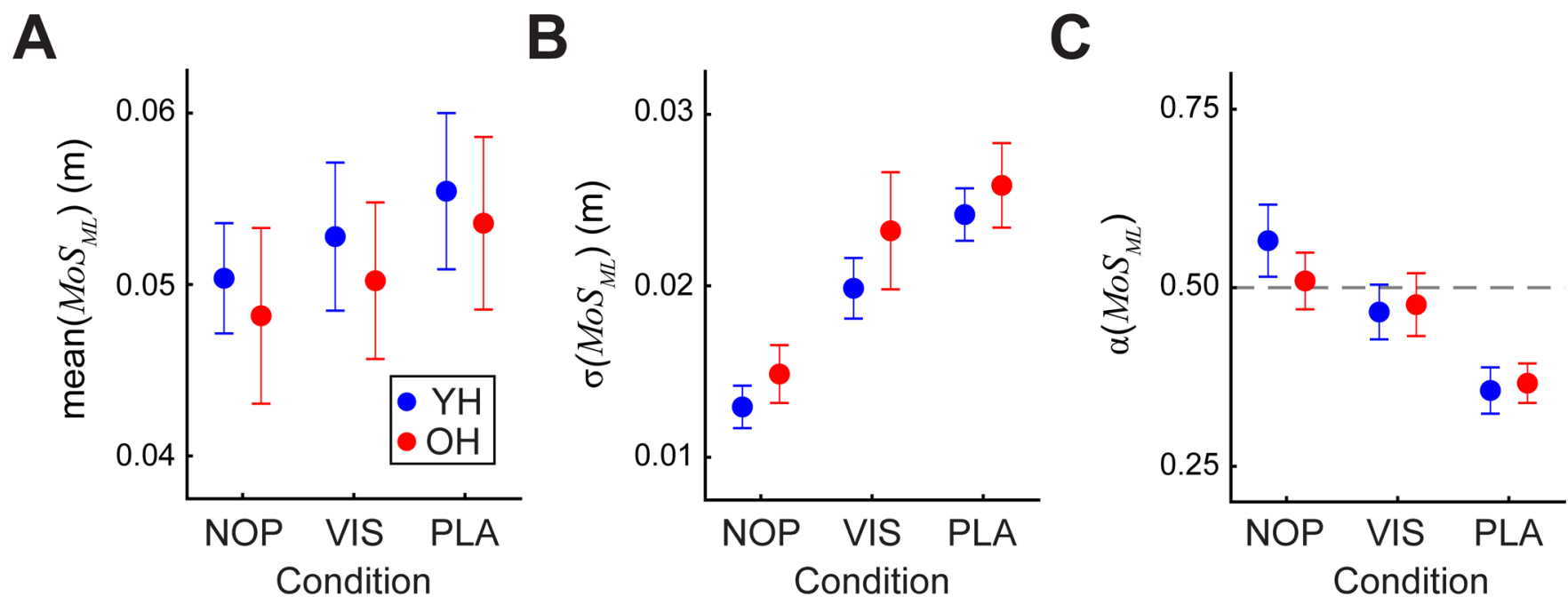

Figure 4: (A) $M o S_{M L}$ mean, (B) variability $\left(\sigma\left(M o S_{M L}\right)\right.$ ) and (C) statistical persistence (DFA exponent: $\left.\alpha\left(M o S_{M L}\right)\right)$ for both YH and $\mathrm{OH}$ age groups for each of NOP, VIS and PLAT conditions. Error bars represent between-participant $\pm 95 \%$ confidence intervals. During VIS and PLAT, participants demonstrated larger $\left(\mathrm{p}=2.83 \times 10^{-12}\right)$, more-variable $\left(\mathrm{p}=1.06 \times 10^{-59}\right)$, and increasingly antipersistent $\left(\mathrm{p}=1.01 \times 10^{-24}\right) M o S_{M L}$, relative to NOP. No significant age group effects were observed (see Supplement, Table S1). 
bioRxiv preprint doi: https://doi.org/10.1101/2021.12.01.470263; this version posted December 3, 2021. The copyright holder for this preprint (which was not certified by peer review) is the author/funder, who has granted bioRxiv a license to display the preprint in perpetuity. It is made

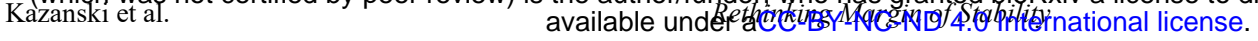

A

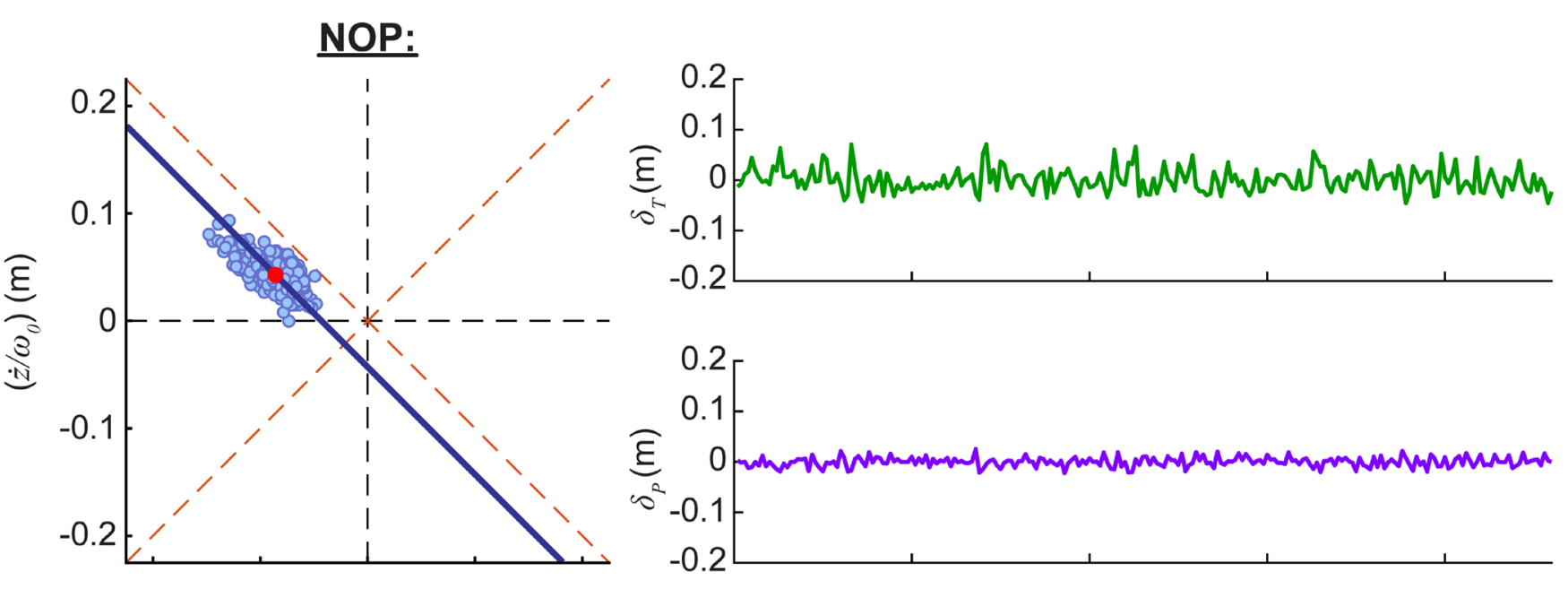

B

\section{VIS:}
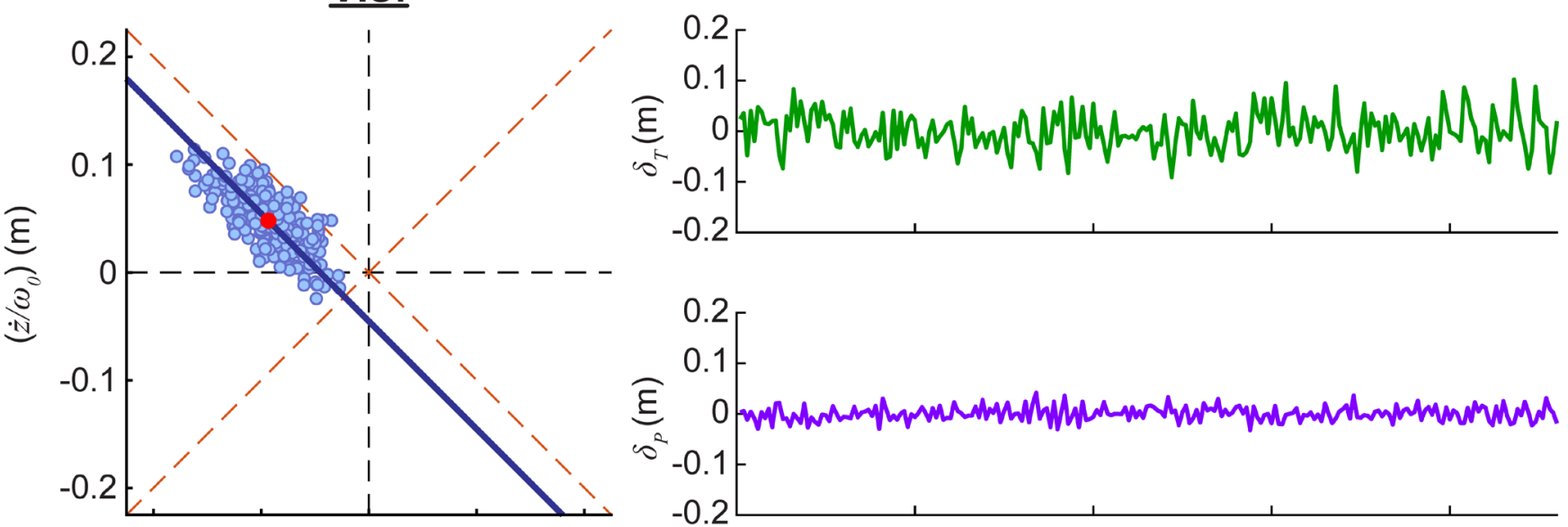

C

PLA:
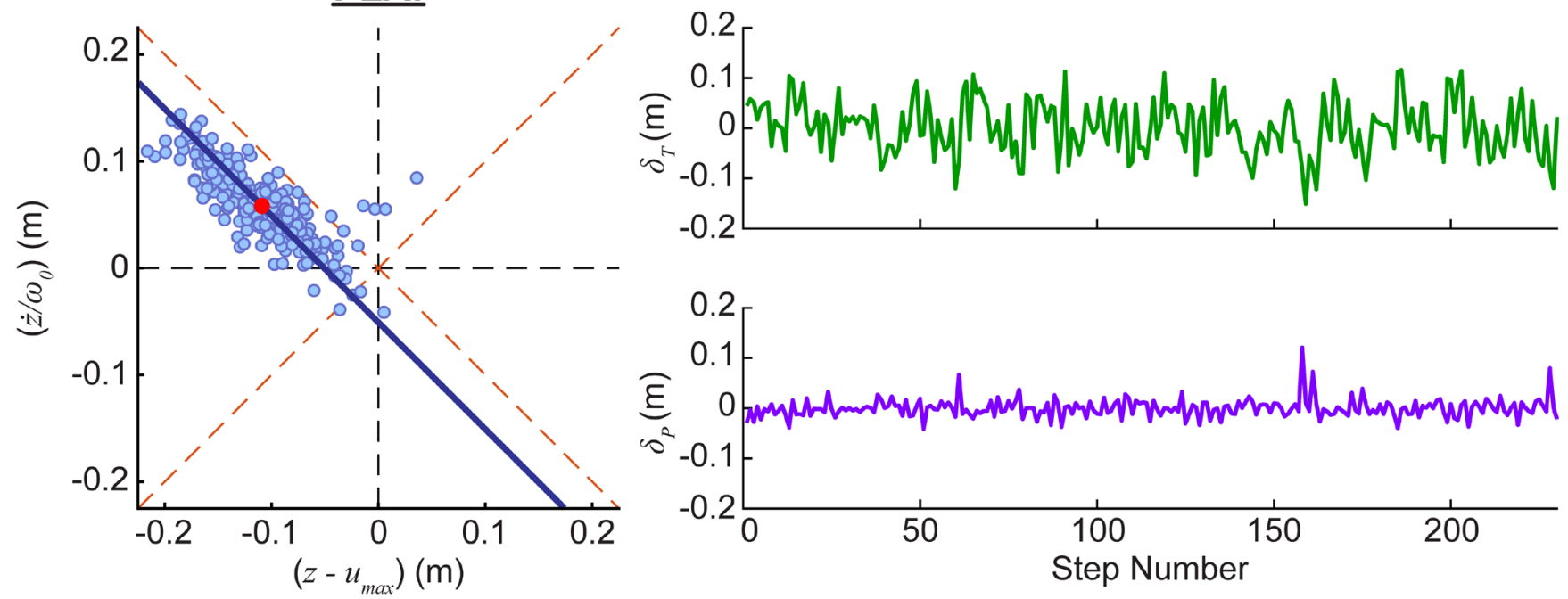

Figure 5 - Example data from representative trials of a typical participant for each of (A) NOP, (B) VIS, and (C) PLAT conditions. Left: Mediolateral CoM dynamics attained at $\left(M o S_{M L}\right)_{n}$ for each $n^{\text {th }}$ step (blue markers) of the corresponding trial. The candidate stability GEM (blue line) is defined by trial-average $M o S_{M L}{ }^{*}$ and the operating point (red marker) is defined by trial-average [( $z$ $\left.u_{\max }\right)^{*},\left(\dot{z} / \omega_{0}\right)^{*}$. Right: Corresponding step-to-step time series of deviations from the operating point, tangent $\left(\delta_{T}\right)$ and perpendicular $\left(\delta_{P}\right)$ to the candidate GEM. 

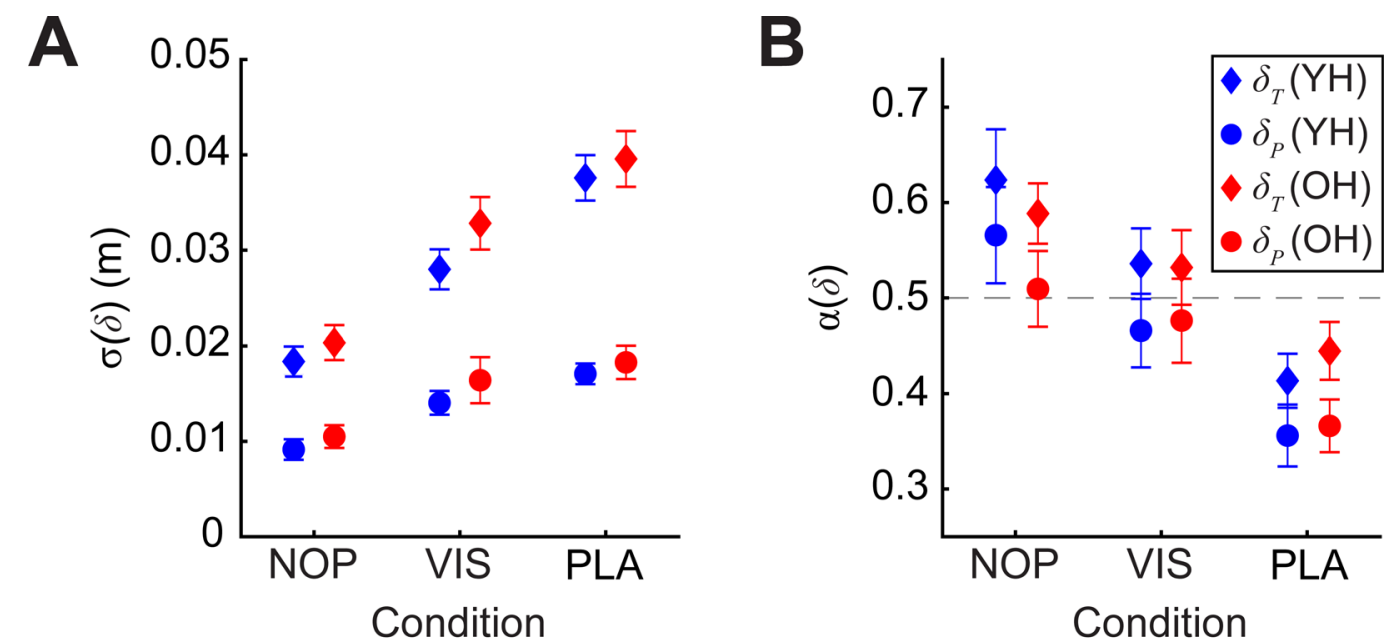

Figure 6: (A): Variability $(\sigma(\delta))$ for all $\delta_{T}$ and $\delta_{P}$ deviation time series for $\mathrm{YH}$ and $\mathrm{OH}$ age groups, for each condition (NOP, VIS and PLA). (B): DFA $\alpha$ exponents for all $\delta_{T}$ and $\delta_{P}$ time series for YH and $\mathrm{OH}$ age groups, for each condition. Error bars represent between-subject $\pm 95 \%$ confidence intervals. Goal-equivalent $\delta_{T}$ deviations were consistently more-variable $(\mathrm{p}=$ $\left.1.10 \times 10^{-103}\right)$ and either more-persistent (higher $\left.\alpha>0.5\right)$ or less anti-persistent (higher $\left.\alpha<0.5\right)\left(\mathrm{p}=7.38 \times 10^{-47}\right)$ than goal-relevant $\delta_{P}$ deviations. During VIS and PLAT perturbations, participants demonstrated more-variable and either less-persistent or antipersistent goal-equivalent $\delta_{T}\left(\mathrm{p}=5.05 \times 10^{-64} ; \mathrm{p}=3.77 \times 10^{-21}\right)$ and goal-relevant $\delta_{P}\left(\mathrm{p}=1.06 \times 10^{-59} ; \mathrm{p}=1.01 \times 10^{-24}\right)$ deviations, relative to NOP. No significant age group effects were observed (see Supplement, Tables S2-S3).

Across age groups and conditions, the extent to which participants regulated step width, $\alpha(w)$, and absolute lateral position, $\alpha\left(z_{B}\right)$, strongly predicted the extent to which step-to-step CoM deviations, $\alpha\left(\delta_{T}\right)$ and $\alpha\left(\delta_{P}\right)$, were subsequently regulated (Fig. 7).

Both YH and $\mathrm{OH}$ participants exhibited increased PoI $_{L}$ (Fig. 8C; all $\mathrm{p}<10^{-9}$; Table S4) when perturbed, indicating a greater likelihood of being laterally unstable for any future step, despite simultaneously exhibiting larger average $M o S_{M L}$ (Fig. 4A). This is due to increased $\delta_{P}$ variance (Fig. 8A-B). While OH demonstrated slightly larger $P o I_{L}$, age group effects were not significant.

A
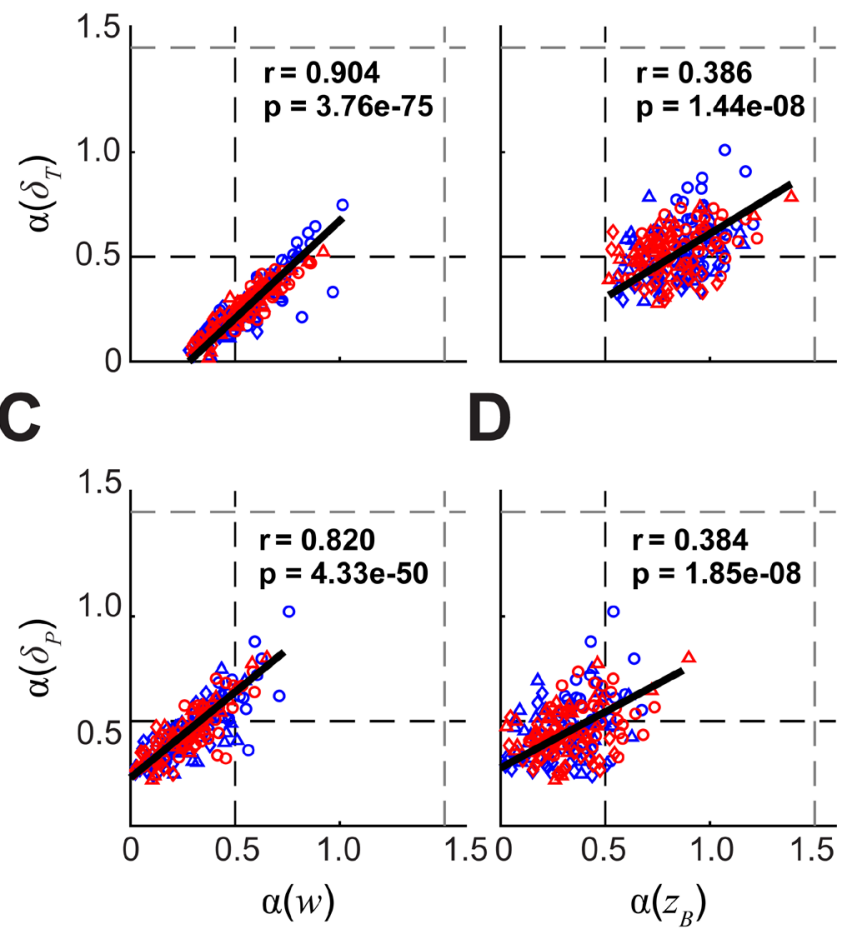

Figure 7: Pairwise Pearson correlations of DFA $\alpha$ exponents for time series of step width ( $w ; \mathbf{A}$ and $\mathbf{C}$ ) and mediolateral body position $\left(z_{B} ; \mathbf{B}\right.$ and $\left.\mathbf{D}\right)$ with those of mediolateral CoM deviations tangent $\left(\delta_{T} ; \mathbf{A}\right.$ and $\left.\mathbf{B}\right)$ and perpendicular $\left(\delta_{P} ; \mathbf{C}\right.$ and D) to the candidate stability GEM. Data were pooled across age groups and conditions. YH adults are visualized with blue markers, $\mathrm{OH}$ adults with red markers. Conditions are depicted as NOP $(\circ)$, VIS $(\Delta)$, PLAT $(\diamond)$. The step-to-step statistical persistence of both $w$ and $z_{B}$ stepping time series significantly predicted that of each of the $\delta_{T}$ and $\delta_{P}$ CoM time series. 
A
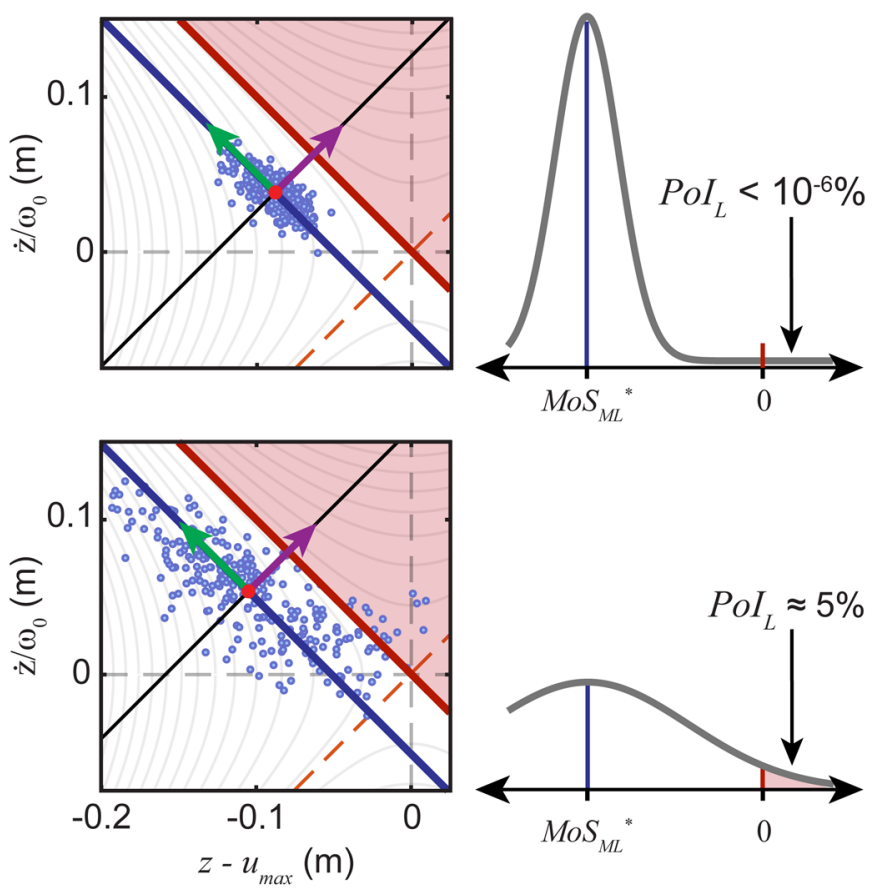

Figure 8: (A): Data from two representative trials (top: YH participant during NOP; bottom: $\mathrm{OH}$ participant during PLA). Both participants demonstrated nearly identical average $M o S_{M L}$ and hence nearly-identical candidate $M o S_{M L}{ }^{*}$ GEMs (left). However, the increased variance in the $\mathrm{OH}$ PLAT trial (bottom) produced substantially greater intrinsic instability likelihood $\left(\mathrm{PoI}_{L}\right.$; right). Thus, average $M o S_{M L}$ alone do not reflect instability likelihood (Fig. 3). (B): Box plots of $\mathrm{PoI}_{L}$ results for all trials for all participants. During VIS and PLAT perturbations, participants demonstrated significantly larger $\operatorname{PoI}_{L}\left(\mathrm{p}=1.08 \times 10^{-34}\right)$, relative to NOP. While some $\mathrm{OH}$ participants demonstrated larger $\mathrm{PoI}_{L}$, group differences were not statistically significant $(\mathrm{p}=0.223)$ (see Supplement, Table S4).

B

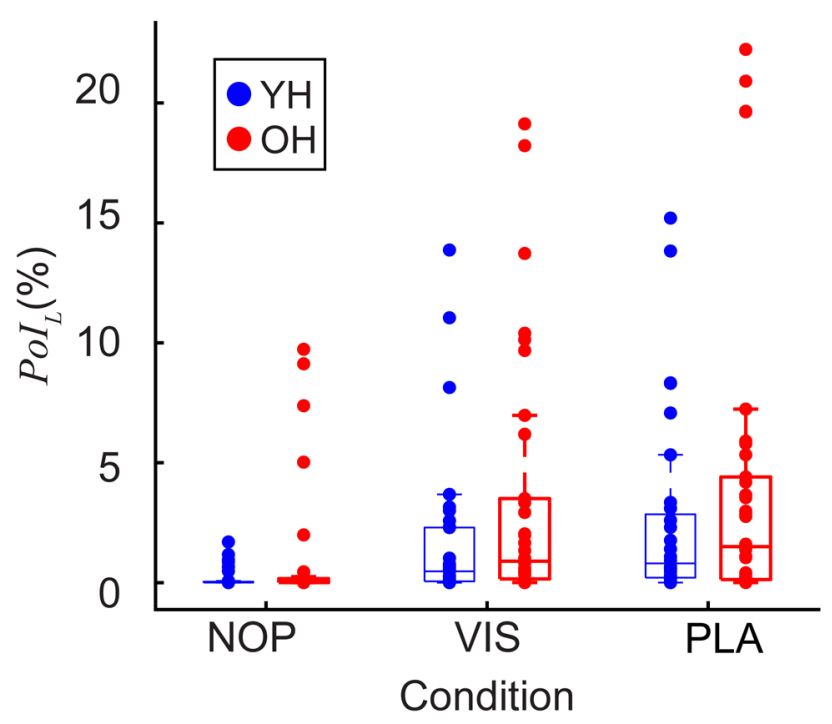

\section{DISCUSSION}

$M o S_{M L}$ (Hof et al., 2005) has been widely-implemented to assess mediolateral balance during human walking. However, when studies average $M o S_{M L}$ across steps, a paradox arises. Gait pathologies (Watson et al., 2021) and destabilizing environments (Wu et al., 2017) induce walkers to exhibit larger average $M o S_{M L}$. Given the fundamental ideas underlying $M o S_{M L}$, these larger values suggest greater mediolateral stability, despite clearly destabilizing intrinsic and/or extrinsic factors. Some studies assume such counterintuitive results reflect a compensatory strategy used to reduce likelihood of balance loss (Madehkhaksar et al., 2018; Onushko et al., 2019). This cannot explain why balanceimpaired and extrinsically-destabilized individuals remain at elevated risk for sideways falls. Thus, before considering whether $M o S_{M L}$ interpretations can even address falling likelihood (Hak et al., 2019; Herssens et al., 2020), we must first resolve this paradox. Others have similarly used phase planes to visualize instantaneous balance dynamics (Bottaro 
et al., 2005) and predict effects of specific within-step corrective actions (Hof, 2008; Reimann et al., 2018). Our work here directly extends these studies to also consider corrective actions enacted across steps.

Averaging $M o S_{M L}$ across steps discards all information pertaining to stepping dynamics. These dynamics provide insights into the control schemes people use to regulate dynamical fluctuations across steps (Dingwell and Cusumano, 2019). Here, we present a unified framework that first defines a constant- $M o S_{M L}$ control hypothesis suggested by both simulated (Hof, 2008) and empirically-observed (Curtze et al., 2011; Rosenblatt and Grabiner, 2010) human walking. We tested this hypothesis by evaluating step-to-step regulation of observed CoM dynamics. Consistent with this hypothesis, participants exhibited goal-relevant $\left(\delta_{P}\right)$ deviations that were far less-variable than goal-equivalent $\left(\delta_{T}\right)$ deviations (Fig. 6A), and readily corrected $\delta_{P}$ deviations off of the constant-MoS $S_{M L}$ GEM (i.e., $\alpha\left(\delta_{P}\right) \approx 0.5$; Fig. 6B). However, they also readily corrected $\delta_{T}$ deviations (i.e., $\alpha\left(\delta_{P}\right)<\alpha\left(\delta_{T}\right)<<1$; Fig. 6B), even though $\delta_{T}$ deviations have no bearing on maintaining constant $M o S_{M L}$. This is not consistent with our hypothesis of constant-MoS $S_{M L}$ control, which would explicitly recognize the GEM and exploit equifinality along it (Dingwell et al., 2010). Thus, we reject this hypothesis and conclude that how humans regulate mediolateral CoM fluctuations from step-to-step involves something more than simply seeking some constant $M o S_{M L}$.

By its very nature, steady locomotion must be carried out and regulated by appropriately executed stepping sequences. Foot placements have long been generally related to mediolateral balance maintenance and, on average, indeed predict average $M o S_{M L}$ responses to mediolateral perturbations (Hof et al., 2010; Li and Huang, 2021). Here, we demonstrate that CoM states comprising $M_{o} S_{M L}$ and relevant mediolateral stepping variables are also regulated from step-to-step in mutually consistent ways. In particular, the extent to which participants regulated foot placement to maintain step width ( $w$ ) (and, to a lesser extent, lateral position, $z_{B}$ ) strongly predicted how both $\delta_{P}$ and $\delta_{T}$ deviations in mediolateral CoM state were corrected (Fig. 7). Because walking humans cannot directly actuate their CoM itself, these results suggest that regulation of the mediolateral CoM state occurs as a biomechanical consequence of how people regulate their foot placements (Kazanski et al., 2020). This idea is consistent with findings that foot placement sets the initial push-off condition (Matthis et al., 2017) for CoM redirection (Joshi and Srinivasan, 2019) and subsequent modulation of $M o S_{M L}$ (Afschrift et al., 2018).

Consistent with the aforementioned paradox (Li and Huang, 2021; McAndrew Young et al., 2012; Onushko et al., 2019), all participants here demonstrated larger average $M o S_{M L}$ when subjected to mediolaterally destabilizing perturbations (Fig. 4A). These average $M o S_{M L}$ can only indicate a possible target value used for stepping regulation, that is, where a candidate stability GEM is located in the mediolateral CoM phase plane (Fig. 2B). Average $M o S_{M L}$ cannot predict the likelihood of becoming unstable on any future step. For this, we must characterize how CoM dynamics are distributed across Stability and Instability regions (Pai and Patton, 1997). Our work is the first to use distribution characteristics across regions of CoM state space to estimate single-step mediolateral instability risk, quantified in the lateral direction as PoI $_{L}$. Consistent with the nature of the experimental destabilizing conditions (VIS and PLAT), PoI $_{L}$ revealed (Fig. 8) that participants' increased variability (Figs. 4B \& 6A) made them more likely to become laterally unstable when perturbed. No participants actually fell during this experiment. Thus, while perhaps more likely to fall, participants successfully recruited active rebalancing mechanisms to counteract these instances of intrinsic lateral instability. Participants exhibited increased instability risk, as indicated by larger $P_{o} I_{L}$ and despite larger average $M o S_{M L}$. From our unified framework addressing balance maintenance via CoM state regulation, PoI emerges as a simple clinical statistic that incorporates step-to-step variance to resolve the present $M o S_{M L}$ paradox. As such, PoI should be used in place of average $M o S_{M L}$ to draw meaningful conclusions regarding mediolateral instability risk. 


\section{ACKNOWLEDGEMENTS}

This work was supported by NIH grant 1-R01-AG049735.

\section{REFERENCES}

Afschrift, M., Pitto, L., Aerts, W., van Deursen, R., Jonkers, I., De Groote, F., 2018. Modulation of gluteus medius activity reflects the potential of the muscle to meet the mechanical demands during perturbed walking. Scientific Reports 8, 11675.

Andrews, L.C., 1998. Special functions of mathematics for engineers. SPIE Press.

Arvin, M., Mazaheri, M., Hoozemans, M.J.M., Pijnappels, M., Burger, B.J., Verschueren, S.M.P., van Dieën, J.H., 2016. Effects of narrow base gait on mediolateral balance control in young and older adults. Journal of Biomechanics 49, 1264-1267.

Bottaro, A., Casadio, M., Morasso, P.G., Sanguineti, V., 2005. Body sway during quiet standing: Is it the residual chattering of an intermittent stabilization process? Human Movement Science 24, 588-615.

Braun, D.A., Nagengast, A.J., Wolpert, D., 2011. Risk-sensitivity in sensorimotor control. Frontiers in Human Neuroscience 5.

Bruijn, S.M., van Dieën, J.H., 2018. Control of human gait stability through foot placement. Journal of The Royal Society Interface 15, 1-11.

CDC, C.f.D.C.a.P., Web-based Injury Statistics Query and Reporting System (WISQARSTM).

Cho, H., Heijnen, M.J.H., Craig, B.A., Rietdyk, S., 2021. Falls in young adults: The effect of sex, physical activity, and prescription medications. PLOS ONE 16, e0250360.

Crenshaw, J.R., Bernhardt, K.A., Achenbach, S.J., Atkinson, E.J., Khosla, S., Kaufman, K.R., Amin, S.T., 2017. The circumstances, orientations, and impact locations of falls in community-dwelling older women. Archives of Gerontology and Geriatrics 73, 240-247.

Curtze, C., Hof, A.L., Postema, K., Otten, B., 2011. Over rough and smooth: Amputee gait on an irregular surface. Gait \& Posture 33, 292-296.

Cusumano, J.P., Cesari, P., 2006. Body-Goal Variability Mapping in an Aiming Task. Biological Cybernetics 94, 367-379.

Day, K.V., Kautz, S.A., Wu, S.S., Suter, S.P., Behrman, A.L., 2012. Foot placement variability as a walking balance mechanism post-spinal cord injury. Clinical Biomechanics 27, 145-150.

Dean, J.C., Alexander, N.B., Kuo, A.D., 2007. The effect of lateral stabilization on walking in young and old adults. IEEE Transactions on Biomedical Engineering 54, 1919-1926.

Dingwell, J.B., Cusumano, J.P., 2010. Re-Interpreting Detrended Fluctuation Analyses of Stride-To-Stride Variability in Human Walking. Gait \& Posture 32, 348-353.

Dingwell, J.B., Cusumano, J.P., 2019. Humans use multi-objective control to regulate lateral foot placement when walking. PLoS Computational Biology 15.

Dingwell, J.B., John, J., Cusumano, J.P., 2010. Do Humans Optimally Exploit Redundancy to Control Step Variability in Walking? PLoS Computational Biology 6, e1000856.

Hak, L., Hettinga, F.J., Duffy, K.R., Jackson, J., Sandercock, G.R.H., Taylor, M.J.D., 2019. The concept of margins of stability can be used to better understand a change in obstacle crossing strategy with an increase in age. Journal of Biomechanics 84, $147-$ 152.

Hausdorff, J.M., Peng, C.K., Ladin, Z., Wei, J.Y., Goldberger, A.L., 1995. Is Walking a Random Walk? Evidence for LongRange Correlations in Stride Interval of Human Gait. Journal of Applied Physiology 78, 349-358. 
Heijnen, M.J.H., Rietdyk, S., 2016. Falls in young adults: Perceived causes and environmental factors assessed with a daily online survey. Human Movement Science 46, 86-95.

Herssens, N., van Criekinge, T., Saeys, W., Truijen, S., Vereeck, L., van Rompaey, V., Hallemans, A., 2020. An investigation of the spatio-temporal parameters of gait and margins of stability throughout adulthood. Journal of the Royal Society Interface 17.

Hof, A.L., 2008. The 'extrapolated center of mass' concept suggests a simple control of balance in walking. Human Movement Science 27, 112-125.

Hof, A.L., Gazendam, M.G.J., Sinke, W.E., 2005. The condition for dynamic stability. Journal of Biomechanics 38, 1-8.

Hof, A.L., van Bockel, R.M., Schoppen, T., Postema, K., 2007. Control of lateral balance in walking: Experimental findings in normal subjects and above-knee amputees. Gait \& Posture 25, 250-258.

Hof, A.L., Vermerris, S.M., Gjaltema, W.A., 2010. Balance responses to lateral perturbations in human treadmill walking. The Journal of Experimental Biology 213, 2655-2664.

Hsieh, K.L., Sheehan, R.C., Wilken, J.M., Dingwell, J.B., 2018. Healthy individuals are more maneuverable when walking slower while navigating a virtual obstacle course. Gait \& Posture 61, 466-472.

John, J., Dingwell, J.B., Cusumano, J.P., 2016. Error Correction and the Structure of Inter-Trial Fluctuations in a Redundant Movement Task. PLoS Computational Biology 12, e1005118.

Joshi, V., Srinivasan, M., 2019. A controller for walking derived from how humans recover from perturbations. J R Soc Interface $16,20190027$.

Kannus, P., Leiponen, P., Parkkari, J., Palvanen, M., Järvinen, M., 2006. A sideways fall and hip fracture. Bone 39, 383-384.

Kazanski, M.E., Dingwell, J.B., Cusumano, J.P., 2020. How healthy older adults regulate lateral foot placement while walking in laterally destabilizing environments. Journal of Biomechanics 104, 109714.

Kuo, A.D., 1999. Stabilization of Lateral Motion in Passive Dynamic Walking. The International Journal of Robotics Research $18,917-930$.

Kuo, A.D., 2007. The six determinants of gait and the inverted pendulum analogy: A dynamic walking perspective. Human Movement Science 26, 617-656.

Li, J., Huang, H.J., 2021. Small Directional Treadmill Perturbations Induce Differential Gait Stability Adaptation. bioRxiv, 2021.2002.2027.433210.

Madehkhaksar, F., Klenk, J., Sczuka, K., Gordt, K., Melzer, I., Schwenk, M., 2018. The effects of unexpected mechanical perturbations during treadmill walking on spatiotemporal gait parameters, and the dynamic stability measures by which to quantify postural response. PLOS ONE 13, e0195902.

Matthis, J.S., Barton, S.L., Fajen, B.R., 2017. The critical phase for visual control of human walking over complex terrain. Proceedings of the National Academy of Sciences 114, E6720-E6729.

McAndrew, P.M., Wilken, J.M., Dingwell, J.B., 2011. Dynamic Stability of Human Walking in Visually and Mechanically Destabilizing Environments. Journal of Biomechanics 44, 644-649.

McAndrew Young, P.M., Wilken, J.M., Dingwell, J.B., 2012. Dynamic margins of stability during human walking in destabilizing environments. Journal of Biomechanics 45, 1053-1059.

McGeer, T., 1990. Passive Dynamic Walking. International Journal of Robotics Research 9, 68-82.

Nagengast, A.J., Braun, D.A., Wolpert, D.M., 2011. Risk-sensitivity and the mean-variance trade-off: decision making in sensorimotor control. Proceedings of the Royal Society B: Biological Sciences 278, 2325-2332. 
Onushko, T., Boerger, T., Van Dehy, J., Schmit, B.D., 2019. Dynamic stability and stepping strategies of young healthy adults walking on an oscillating treadmill. PLoS One 14, e0212207.

Otten, E., 1999. Balancing on a Narrow Ridge: Biomechanics and Control. Philosophical Transactions of the Royal Society B: Biological Sciences 354, 869-875.

Pai, Y.C., Patton, J.L., 1997. Center of Mass Velocity-Position Predictions for Balance Control. Journal of Biomechanics 30, 347-354.

Patil, N.S., Dingwell, J., Cusumano, J.P., 2019. Correlations of pelvis state to foot placement do not imply within-stepactive control. Journal of Biomechanics 97, 109375.

Peebles, A.T., Reinholdt, A., Bruetsch, A.P., Lynch, S.G., Huisinga, J.M., 2016. Dynamic margin of stability during gait is altered in persons with multiple sclerosis. J Biomech 49, 3949-3955.

Reimann, H., Fettrow, T.D., Jeka, J.J., 2018. Strategies for the Control of Balance During Locomotion. Kinesiology Review 7, $18-25$.

Render, A.C., Kazanski, M.E., Cusumano, J.P., Dingwell, J.B., 2021. Walking humans trade off different task goals to regulate lateral stepping. Journal of Biomechanics 119, 110314.

Rosenblatt, N.J., Grabiner, M.D., 2010. Measures of frontal plane stability during treadmill and overground walking. Gait \& Posture 31, 380-384.

Selgrade, B.P., Childs, M.E., Franz, J.R., 2020. Effects of aging and target location on reaction time and accuracy of lateral precision stepping during walking. J Biomech 104, 109710.

Tisserand, R., Armand, S., Allali, G., Schnider, A., Baillieul, S., 2018. Cognitive-motor dual-task interference modulates mediolateral dynamic stability during gait in post-stroke individuals. Human Movement Science 58, 175-184.

Wang, Y., Srinivasan, M., 2014. Stepping in the direction of the fall: the next foot placement can be predicted from current upper body state in steady-state walking. Biology Letters 10, 20140405.

Watson, F., Fino, P.C., Thornton, M., Heracleous, C., Loureiro, R., Leong, J.J.H., 2021. Use of the margin of stability to quantify stability in pathologic gait - a qualitative systematic review. BMC Musculoskeletal Disorders 22, 597.

Winter, D.A., 1995. Human Balance And Posture Control During Standing And Walking. Gait \& Posture 3, 193-214.

Wu, M.M., Brown, G., Gordon, K.E., 2017. Control of locomotor stability in stabilizing and destabilizing environments. Gait \& Posture 55, 191-198.

Yang, Y., Komisar, V., Shishov, N., Lo, B., Korall, A.M., Feldman, F., Robinovitch, S.N., 2020. The Effect of Fall Biomechanics on Risk for Hip Fracture in Older Adults: A Cohort Study of Video-Captured Falls in Long-Term Care. J Bone Miner Res 35, 1914-1922.

Zeni, J.A., Richards, J.G., Higginson, J.S., 2008. Two simple methods for determining gait events during treadmill and overground walking using kinematic data. Gait \& Posture 27, 710-714. 